Dhaka Univ. J. Biol. Sci. 25(1): 39-46, 2016 (January)

\title{
ABUNDANCE OF HELMINTH PARASITE IN CHANNA STRIATUS (BLOCH, 1793) FROM PUNARBHABA AND ATRAI RIVERS
}

\author{
Sujon Kundu And Aminul I. BhuiYAN* \\ Department of Zoology, University of Dhaka, Dhaka 1000, Bangladesh \\ Key words: Helminth parasites, Abundance, Channa striatus
}

\begin{abstract}
The study was conducted to determine the impact of seasons, host size and sexes on the incidence of helminth community infecting Channa striatus (Bloch, 1793) from Punarbhaba and Atrai rivers of Dinajpur district. Four helminths were identified: one Cestoda, Bothriocephalus cuspidatus (Cooper, 1917); two Nematoda, Ascaridia spp. (Dujardin, 1845) and Camallanus intestinalus (Bashirullah, 1974); one Acanthocephala, Pallisentis ophiocephali (Thapar, 1930). Out of 75 hosts examined 52 were infected by 326 helminth parasites. Nematoda was the most dominant group (48.0\%), followed by Acanthocephala (46.67\%) and Cestoda (8.0\%). The larger hosts were more infected than the smaller ones. A significant positive correlation was observed between the length of the host with respect to the abundance and prevalence of parasite for $C$. intentinalus and $P$. ophiocephali. Regarding the effect of seasonal changes, the prevalence of $B$. cuspidatus (14.81\%) and Ascaridia spp. (22.22\%) was highest in the rainy season. The highest prevalence of $C$. intestinalus $(73.91 \%)$ was observed in winter. The prevalence of $P$. ophiocephali was high $(84.0 \%)$ in summer. Except the abundance of B. cuspidatus $(\mathrm{p}=0.147)$ all parasite species, Ascaridia spp. $(\mathrm{p}=0.003), C$. intestinalus $(\mathrm{p}<0.01)$ and P. ophiocephali $(\mathrm{p}<0.01)$ varied significantly among seasons. Overall prevalence of infection of male and female were $53.8 \%$ and $77.6 \%$, respectively. A significant difference in the abundance of the parasite species with respect to host sex was observed only in C. intestinalus $(Z=-2.886$, $\mathrm{p}=0.004)$.
\end{abstract}

\section{Introduction}

Any parasite living in or on a fish, extent some degree of harmful influence on its host thus normal growth of fish is interrupted or inhibited if they are heavily infested with parasites. Besides the feeding behaviour and habitat selection by the host fish, the key factors of parasitic infection are host species, age, size, sex, immunological status, and environmental changes ${ }^{(1)}$. Predatory fish expose more infective helminth larvae in their diet than planktivores. After over evolutionary time, it translates into higher parasite colonization rates in predatory fish than in planktivorous fish ${ }^{(2)}$. The major parasitic groups found in freshwater fishes are trematodes (monogeneas and digeneans), cestodes, nematodes and acanthocephalans that complete their life cycles through intermediate hosts ${ }^{(3)}$.

*Author for correspondence: <aminul_zool@du.ac.bd> 
A few work on parasites of C. striatus have been done in Bangladesh ${ }^{(4-7)}$. In the earlier research on parasites of $C$. striatus, most of the work were done on the fish of around Dhaka, Mymensingh and southern part of the country. In most of the cases the examined fish were collected from the local markets, where there is a chance of mixing fish from different areas and this may affect the correct information about source of the host fish. During the present study fish were collected from the certain areas of Dinajpur district, northern part of Bangladesh directly from people who caught them. So this sample will represent more correct information of the location of the host fish, C. striatus.

The present work was undertaken to investigate the abundance of helminth parasites with regard to season, sex and length of host fish $C$. striatus collected from the two rivers of Dinajpur district.

\section{Materials and Methods}

A total of 75 Channa striatus were collected from Punarbhaba River $\left(2^{\circ} 33^{\prime} 39.07^{\prime \prime} \mathrm{N}\right.$,

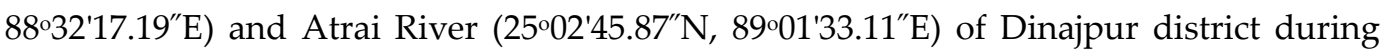
August, 2014 to May, 2015. The frozen fish were transported in an ice-cooled box and live fish in buckets filled with water from the river to the laboratory without spoiling. The collected fish were kept in a refrigerator in the Fisheries laboratory, Department of Zoology, University of Dhaka, before autopsy. To observe the seasonal trend of helminth infestation, whole year was divided into three seasons arbiturely: Rainy season - August to October; Winter - November to January, and Summer - March to May. The sample sizes were 27, 23 and 25, respectively. Length, sex and organ wise infections were recorded. Length and sex of fish were noted before autopsy. Extensive search was made for helminth parasites infecting the fish using Lica Zoom 2000 Dissecting Microscope. All the parasites from each organ were sorted, cleaned, counted and then preserved in $70 \%$ alcohol. The cestodes were stained in acetic carmine for 2 - 3 hours. After staining, the worms were washed in $70 \%$ alcohol to which a few drops of glacial acetic acid were added. Then they were cleaned and temporarily mounted in lactophenol. Nematodes were cleaned and temporarily mounted in lactophenol for microscopic study. Berland's method(8) was used for dehydration and mounting. One nematode, Ascaridia could not be identified to the species level, therefore referred to as Ascraidia spp. Parasite identification was done using taxonomic keys from Yamaguti ${ }^{(9-11)}$. Terminologies used in the studies as proposed by Margolis et al. ${ }^{(12)}$.

Standard statistical computations were carried out using SPSS 20 software. Correlation between the host length with the abundance and prevalence of parasites were observed by determining Pearson's linear correlation coefficient (r) and Spearman's rank correlation coefficient $\left(r_{s}\right)$. A Kruskal-Wallis $(\mathrm{K}-\mathrm{W})$ test was applied to find significant differences in the abundance of the parasite species for seasons. The Mann- 
Whitney $(\mathrm{M}-\mathrm{W}) \mathrm{U}$ test was used to measure the correlation between the abundance of each helminth species and the host's sex.

\section{Results and Discussion}

During the study period from August 2014 to May 2015, a total of 75 fishes were examined for the abundance and prevalence of helminth parasites. Four species of metazoan parasites (1 Cestoda, 2 Nematoda and 1 Acanthocephala) were collected mostly from the intestine of the host fish. The total number of parasite individuals were 326, among them 15 were cestodes, 180 nematodes and 131 acanthocephalans.

The prevalence of individual species of helminth showed a steady increase with host length (Table 1). The highest prevalence of B. cuspidatus $(17.86 \%)$ and C. intestinalus $(57.14 \%)$ was found in length group $32-36 \mathrm{~cm}$, for Ascaridia spp. (18.52\%) and $P$. ophiocephali (51.85\%) in fish of 27 - $31 \mathrm{~cm}$ compared with other size classes. The highest mean intensity and abundance of all the four parasites were found in $32.0-36.0 \mathrm{~cm}$ length group of fish. A positive relationship between the level of parasitic infection and the size of the host fish have been reported ${ }^{(4)}$. Large fish provide more internal space for parasite establishment and incur higher infection rates as they eat more parasitized prey $^{(13)}$. This observation agrees with the finding of Ghani and Bhuiyan ${ }^{(7)}$. They reported that the hosts of bigger length were found to be more infected than the smaller ones. This might be due to the fact that the bigger ones cover wider areas in search of food. As a result, large fish takes more food than the smaller ones and this exposed them to infection by more parasites.

According to Pearson's coefficient correlation ( $r$ ) and Spearman's rank correlation coefficient $\left(\mathrm{r}_{\mathrm{s}}\right)$, a significant positive correlation was observed between the host length with respect to the abundance and prevalence of Camallanus intestinalus ( $\mathrm{r}=0.235$, $\left.\mathrm{p}<0.05 ; \mathrm{r}_{\mathrm{s}}=0.248, \mathrm{p}<0.05\right)$ and P. ophiocephali $\left(\mathrm{r}=0.285, \mathrm{p}<0.05 ; \mathrm{r}_{\mathrm{s}}=0.243, \mathrm{p}<0.05\right)$ (Table 2). Ascaridia spp. $(\mathrm{r}=-0.094, \mathrm{p}>0.05)$ showed a negative correlation between the host length and abundance of parasite but not significant. B. cuspidatus showed positive correlation between the host length and abundance, prevalence of parasite but not significant $(\mathrm{p}>0.05)$ (Table 2).

The highest prevalence of B. cuspidatus (14.81\%) and Ascaridia spp. (22.22\%) was exhibited from July to October, i.e, in the rainy season. The highest prevalence of $C$. intestinalus (73.91\%) was recorded from November to January, the winter season and that of $P$. ophiocephali (84.0\%) from March to May, the summer season. Overall mean intensity of B. cuspidatus was same, $2.50(( \pm 0.39)$ and $2.50( \pm 0.74)$ ( in rainy season and winter season, respectively. For $C$. intestinalus the mean intensity varied from $2.0( \pm 0.92)$ ( to $5.58( \pm 3.68)$ in the three seasons, the highest mean intensity was observed in winter season. The mean intensity of $P$. ophiocephali was high (4.86) in summer season. The abundance of B. cuspidatus was high (0.37) in rainy season, C. intestinalus (4.13) in winter 
season and P. ophiocephali (4.08) in summer season (Table 3). Bhure and Nanware ${ }^{(14)}$ found high incidence of infection of Senga sp. (Cestode), Gangesia sp. (Trematode), Proteocephalus sp. (Cestode) in summer season followed by winter season whereas infection was low in monsoon season. He indicated that environmental factors and feeding habit influence the seasonality of parasitic infection either directly or indirectly.

Table 1. Prevalence, mean intensity and abundance of helminth infection of $C$. striatus in relation to size of the host fish.

\begin{tabular}{|c|c|c|c|c|}
\hline Size classes & $\mathrm{I}(\mathrm{n}=8)$ & II $(n=12)$ & III (n = 27) & $\mathrm{IV}(\mathrm{n}=28)$ \\
\hline Length $(\mathrm{cm})$ group & $17.0-21.0$ & $22.0-26.0$ & $27.0-31.0$ & $32.0-36.0$ \\
\hline \multicolumn{5}{|l|}{ B. cuspidatus } \\
\hline No. of parasite found & - & - & 2 & 13 \\
\hline Prevalence & - & - & 3.7 & 17.86 \\
\hline Mean intensity $( \pm S D)$ & - & - & $2.0( \pm 0.39)$ & $2.6( \pm 1.04)$ \\
\hline Abundance & - & - & 0.07 & 0.46 \\
\hline \multicolumn{5}{|l|}{ Ascaridia spp. } \\
\hline No. of parasite found & - & 1 & 5 & 9 \\
\hline Prevalence & - & 8.33 & 18.52 & 10.71 \\
\hline Mean intensity $( \pm S D)$ & - & $1( \pm 0.29)$ & $2.5( \pm 0.68)$ & $3.0( \pm 0.74)$ \\
\hline Abundance & - & 0.08 & 0.19 & 0.32 \\
\hline \multicolumn{5}{|l|}{ C. intestinalus } \\
\hline No. of parasite found & 1 & 9 & 65 & 90 \\
\hline Prevalence & 12.5 & 33.33 & 51.85 & 57.14 \\
\hline Mean intensity $( \pm$ SD) & $1.0( \pm 0.36)$ & $2.25( \pm 1.29)$ & $4.64( \pm 3.43)$ & $5.63( \pm 4.02)$ \\
\hline Abundance & 0.13 & 0.75 & 2.41 & 3.21 \\
\hline \multicolumn{5}{|l|}{ P. ophiocephali } \\
\hline No. of parasite found & 2 & 11 & 46 & 72 \\
\hline Prevalence & 12.5 & 50.0 & 51.85 & 50.0 \\
\hline Mean intensity $( \pm S D)$ & $2.0( \pm 0.71)$ & $1.83( \pm 1.08)$ & $3.29( \pm 2.22)$ & $5.14( \pm 2.53)$ \\
\hline Abundance & 0.25 & 0.92 & 1.70 & 2.57 \\
\hline
\end{tabular}

Kaur(15) reported that the cestode exhibited a clear seasonality, with maximum prevalence during summer season of the year due to feeding habit and availability of food. From present study, the maximum infection in summer is probably due to higher evapo-transpiration rate leading to reduced water volume, habitat contraction and higher 
host and parasite densities. Consequently, more contact is made between the host and the parasite, this might be the major factor in parasite transmission. Except the abundance of B. cuspidatus ( $\mathrm{p}=0.147$ ) all the parasite species (Ascaridia spp. $\mathrm{p}=0.003, \mathrm{C}$. intestinalus $\mathrm{p}<0.01$ and $P$. ophiocephali $\mathrm{p}<0.01)$ varied significantly among seasons according to Kruskal-Wallis test $\left(\chi^{2}\right)$ (Table 4).

Table 2. Values of Pearson's correlation coefficients ( $r$ ) and Spearman's correlation coefficients $\left(r_{s}\right)$ to show the relationship between abundance and prevalence with the length of $C$. striatus.

\begin{tabular}{lcccc}
\hline Parasites & $\mathrm{r}$ & $\mathrm{P}$ & $\mathrm{r}_{\mathrm{s}}$ & $\mathrm{p}$ \\
\hline B. cuspidatus & 0.074 & 0.527 & 0.213 & 0.067 \\
Ascaridia spp. & -0.094 & 0.420 & 0.036 & 0.762 \\
C. intestinalus & 0.235 & $0.043^{*}$ & 0.248 & $0.032^{*}$ \\
P. ophiocephali & 0.285 & $0.013^{*}$ & 0.243 & $0.036^{*}$ \\
\hline
\end{tabular}

*Significant level $<0.05$

Table 3. Prevalence, mean intensity and abundance of helminth infection of $C$. striatus according to season.

\begin{tabular}{lllll}
\hline & B. cuspidatus & Ascaridia spp. & C. intestinalus & P. ophiocephali \\
\hline Rainy season & & & & \\
No. of parasite found & 10 & 15 & 58 & 12 \\
Prevalence & 14.81 & 22.22 & 44.44 & 25.93 \\
Mean intensity $( \pm$ SD) & $2.50( \pm 0.93)$ & $2.50( \pm 1.16)$ & $4.83( \pm 3.80)$ & $1.71( \pm 0.80)$ \\
Abundance & 0.37 & 0.56 & 2.18 & 0.44 \\
Winter season & & & & \\
No. of parasite found & 5 & - & 95 & 17 \\
Prevalence & 8.70 & - & 73.91 & 30.40 \\
Mean intensity $( \pm$ SD) & $2.50( \pm 0.74)$ & - & $5.58( \pm 3.68)$ & $2.43( \pm 1.48)$ \\
Abundance & 0.22 & - & 4.13 & 0.74 \\
Summer season & & - & & 102 \\
No. of parasite found & - & - & 24.0 & 84.0 \\
Prevalence & - & - & $2.0( \pm 0.92)$ & $4.86( \pm 2.91)$ \\
Mean intensity $( \pm$ SD) & - & - & 0.48 & 4.08 \\
Abundance & - & & & \\
\hline
\end{tabular}

B. cuspidatus was not found to infect in male. The prevalence, mean intensity and abundance were same to be $12.24 \%, 2.50$ ( \pm 0.85 ) and 0.31 consequently for Ascaridia spp. as those were for B. cuspidatus in female fish. Both prevalence and intensity of $C$. intestinalus and P. ophiocephali were relatively high in female than in male (Table 5). The 
abundance of $C$. intestinalus (3.10) and $P$. ophiocephali (2.31) were high in female than in male host (Table 5). Ghani and Bhuiyan ${ }^{(14)}$ reported higher helminth infection in female than in male host, $C$. punctatus. They also explained that this might be the result of the physiological state of the females. Gravid females had reduced resistance to infection by parasites. The rate of food taken by female for the development of their eggs, might expose them to contact with the parasite. Records on higher prevalence in female hosts is also supported by Gupta et al.(16) who observed that the prevalence of Pallisentis in C. striatus was higher in females as compared to males. In case of C. punctatus, overall prevalence of Pallisentis was higher in males as compared to females. Similarly, in the present study, the prevalence of $P$. ophiocephali in $C$. striatus was higher in females as compared to males.

Differences in the infection status in sexes in present observation might be due to physiological, morphological and behavioral differences between host sex, which could lead to one sex being more prone to parasitic infection than the other.

Table 4. Values of the Kruskal-Wallis test $\left(\chi^{2}\right)$ to find significant differences in the abundance of the parasite species in seasons.

\begin{tabular}{lcl}
\hline Parasites & $\mathrm{K}-\mathrm{W} \chi^{2}$ & $\mathrm{P}$ \\
\hline B. cuspidatus & 3.834 & 0.147 \\
Ascaridia spp. & 11.415 & $0.003^{*}$ \\
C. intestinalus & 16.627 & $0.000^{*}$ \\
P. ophiocephali & 29.933 & $0.000^{*}$ \\
\hline
\end{tabular}

* Significant level $<0.01$

Table 5. Prevalence, mean intensity and abundance of helminth infection of $C$. striatus in relation to host sex.

\begin{tabular}{lllll}
\hline Sex & B. cuspidatus & Ascaridia spp. & C. intestinalus & P. ophiocephali \\
\hline Male & & & & \\
No. of parasite found & - & - & 13 & 18 \\
Prevalence & - & - & 30.77 & 42.31 \\
Mean intensity $( \pm$ SD) & - & - & $1.63( \pm 0.86)$ & $1.64( \pm 0.93)$ \\
Abundance & - & - & 0.50 & 0.69 \\
\hline Female & & & & \\
No. of parasite found & 15 & 15 & 152 & 113 \\
Prevalence & 12.24 & 12.24 & 53.06 & 48.94 \\
Mean intensity $( \pm$ SD) & $2.50( \pm 0.85)$ & $2.50( \pm 0.90)$ & $5.85( \pm 3.87)$ & $4.71( \pm 2.92)$ \\
Abundance & 0.31 & 0.31 & 3.10 & 2.31 \\
\hline
\end{tabular}


Results of Mann-Whitney test showed a significant difference in the abundance of the parasite species with respect to host sex was observed only for $C$. intestinalus $(Z=-2.886$, $\mathrm{p}=0.004$ ) (Table 6). But no significant difference in the abundance of the parasite species with respect to host sex for B. cuspidatus $(Z=-1.846, \mathrm{p}=0.065)$, Ascaridia spp. $(\mathrm{Z}=-1.846$, $\mathrm{p}=0.065)$ and $P$. ophiocephali $(\mathrm{Z}=-1.696, \mathrm{p}=0.090)$ was observed (Table 6).

Table 6. Values of the Mann-Whitney test to find significant differences in the abundance of the parasite species in host sex.

\begin{tabular}{lcl}
\hline Parasites & $\mathrm{Z}$ & $\mathrm{P}$ \\
\hline B. cuspidatus & -1.846 & 0.065 \\
Ascaridia spp. & -1.846 & 0.065 \\
C. intestinalus & -2.886 & $0.004^{*}$ \\
P. ophiocephali & -1.696 & 0.090 \\
\hline
\end{tabular}

*Significant level $<0.01$

The changes in the prevalence and mean intensity of parasitic infection might be influenced by various factors such as water temperature, water pollution, parasite biology, host age, host sex, feeding habits of the host and the availability of infected intermediate hosts. These factors might be interrelated and influenced by each other.

\section{References}

1. Khan RA 2012. Host-parasite interactions in some fish species. J. Parasitol. Research 7, Article ID 237280.

2. Luque JL and R Poulin 2004. Use of fish as intermediate hosts by helminth parasites: A comparative analysis. Acta Parasitologica 49(4): 353-361.

3. Schmidt GD 1990. Essentials of Parasitology (4 ${ }^{\text {th }}$ ed.). W.M.C. Brown Publishers, Texas, USA.

4. Bashirullah AKM 1973. A brief survey of the helminth fauna of certain marine and fresh water fishes of Bangladesh. Bangladesh J. Zool. 1(1): 63-81.

5. Ahmed ATA 1981. Helminth infection in freshwater fishes of Bangladesh. Fish Pathology 15 (3/4): 229-236.

6. Arthur JR and Ahmed ATA 2002. Checklist of the parasites of fishes of Bangladesh. FAO, FTP 369/1: 77.

7. Ghani MO and AI Bhuiyan 2011. Community structures of endoparasitic helminths of Channa punctatus from a freshwater river and a polluated lagoon of Bangladesh. Bangladesh J. Zool. 39(2): 173-185.

8. Berland, B. 1982. Basic techniques involved in helminth preservation. In: Proc. of workshop on "Technology as applied to museum parasite collections". ICOPA V-1982. Toronto, Canada. pp. 1-15.

9. Yamaguti S 1961. Systema Helminthum. Vol. III. The Nematodes of Vertebrates. Interscience Publishers Inc., New York. pp. 1- 423. 
10. Yamaguti S 1959. Systema Helminthum. Vol. II. The Cestodes of vertebrates. Interscience Publishers Inc., New York. pp. 1-860.

11. Yamaguti S 1963. Systema Helminthum. Acanthocephala. Int. Book \& Periodicals Supply Service, New Delhi, India.

12. Margolis L, GW Esch, JC Holmes, AM Kuris and GA Schad 1982. The use of ecological terms in parasitology (report of an ad hoc committee of the American Society of Parasitologists). J. Parasitol. 68: 131-133.

13. Poulin R 2000. Variation in the intraspecific relationship between fish length and intensity of parasitic infection: Biological and statistical causes. J. Fish Biology 56: 123-137.

14. Bhure DB and SS Nanware 2014. Studies on prevalence of cestode parasites of freshwater fish, Channa punctatus. J. Entomol. and Zool. Studies 22(4): 283-285.

15. Kaur P, R Shrivastav, TA Qureshi, S Manohar, JD Saroch and S Sharma 2012. Host parasite relationship: Senga sp. (Dollfus, 1934) in association with naturally infected freshwater host fish Channa punctatus (B1.). J. Chemical, Biological and Physical Sciences 2(4): 21752181.

16. Gupta N, P Singhal and DK Gupta 2012. Population dynamics of a parasite Pallisentis in two species of fish Channa punctatus and Channa striatus. J. Environ. Biol. 33: 195-199.

(Manuscript received on 16 September, 2015; revised on 23 December, 2015) 